\title{
Removal of intraosseous hemangioma in frontal bone under direct vision through a small incision
}

\author{
Hyeon Seok Kim, \\ Woo Seob Kim, \\ Han Koo Kim, \\ Tae Hui Bae \\ Department of Plastic and \\ Reconstructive Surgery, Chung-Ang \\ University Hospital, Seoul, Korea
}

\begin{abstract}
Complete surgical excision within a margin of normal healthy bone is the treatment of choice for intraosseous hemangioma. A 56-year-old man visited with complaints of a firm, mildly tender, immovable, and palpable mass on the right forehead (size: $1.5 \times 1.5 \mathrm{~cm}$ ). Non-contrast brain computed tomography performed preoperatively revealed a $1.5 \mathrm{~cm}$ heterogenous osteolytic lesion with suspected internal trabeculation in the right frontal bone. Under general anesthesia, a $2 \mathrm{~cm}$ transverse incision was made on the forehead skin rather than bicoronal incision. Full-thickness en bloc resection of the frontal bone including the mass was performed. The frontal bone was removed with care taken not to damage the frontal sinus mucosa. The frontal sinus was sealed with a collagen patch (Tachocomb) and a cranioplasty was performed using bone cement. At 6 months postoperative, a clean wound was confirmed without any complications, and there was no local recurrence. Surgical excision of intraosseous hemangioma in the frontal sinus bone can be performed via direct incision or the bicoronal approach. In this case, the direct incision approach was used to achieve smaller scars and faster recovery than the bicoronal approach.
\end{abstract}

Keywords: Frontal bone / Hemangioma / Intraosseous / Surgical wound

\section{INTRODUCTION}

Intraosseous hemangioma is a rare vascular malformation of bone that accounts for $0.7 \%-1.0 \%$ of all bone tumors $[1,2]$. Intraosseous hemangioma usually occurs in the vertebral column or, rarely, in the calvarium; in the latter, it occurs in the parietal bone, followed by the frontal bone [3]. The cause of intraosseous hemangioma has not yet been clarified, but it is reportedly related to previous trauma history $[3,4]$.

Complete surgical excision within a margin of normal healthy bone is the treatment of choice for intraosseous hemangioma. However, complete bone tumor resection in the upper and middle facial area requires a long incision such as a bicoronal incision and immediate reconstruction with bone grafts or allo-

\section{Correspondence: Tae Hui Bae}

Department of Plastic and Reconstructive Surgery, Chung-Ang University

Hospital, 102 Heukseok-ro, Dongjak-gu, Seoul 06973, Korea

E-mail: psbth@cau.ac.kr

Received November 17, 2020 / Revised January 3, 2021 / Accepted February 5, 2021 plastic implants [5].

Here, we describe a case of intraosseous hemangioma surgically treated via a small forehead direct skin incision in the frontal sinus bone rather than a long bicoronal incision.

\section{CASE REPORT}

A 56-year-old man visited with complaints of a firm, mildly tender, immovable, and palpable mass on the right forehead (size: $1.5 \times 1.5 \mathrm{~cm}$ ) that developed 1 year before the visit. The patient reported injuring his right forehead before the symptoms first occurred. Non-contrast brain computed tomography (CT) performed preoperatively revealed a $1.5 \mathrm{~cm}$ heterogenous osteolytic lesion with suspected internal trabeculation in the right frontal bone (Fig. 1). We planned to create a small $2 \mathrm{~cm}$ incision directly above the heterogenous osteolytic mass to remove it. Under general anesthesia, a $2 \mathrm{~cm}$ transverse incision was made on the forehead skin. The frontalis muscle was split 


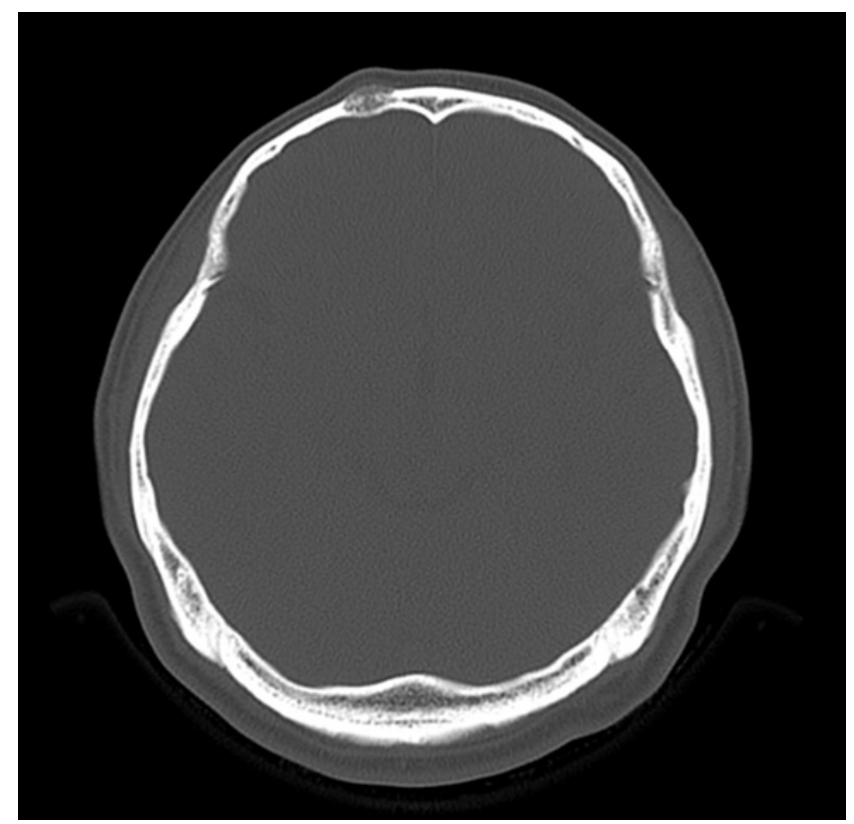

Fig. 1. Preoperative computed tomography image.
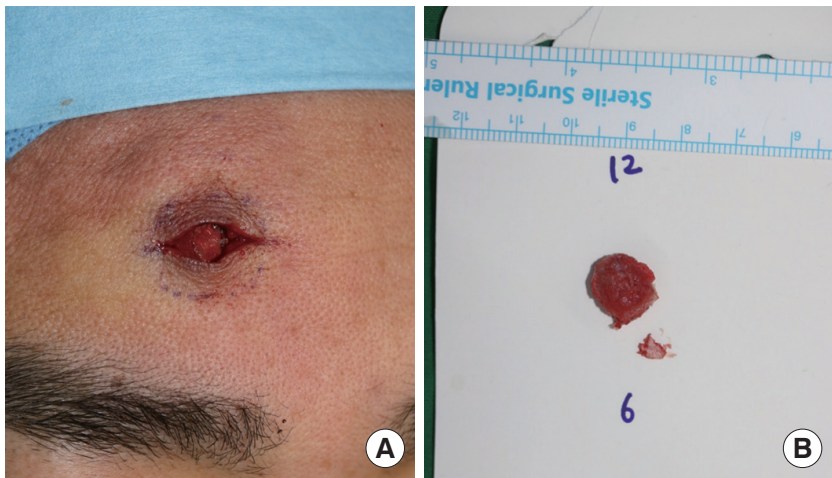

Fig. 2. Intraoperative photographs. (A) Exposed intraosseous hemangioma on the right frontal sinus. (B) Removed intraosseous hemangioma specimen.

vertically and the mass was exposed (Fig. 2A). Several holes were drilled using a cutting burr $2 \mathrm{~mm}$ apart around the mass and connected to each other to expose the dura mater.

Full-thickness en bloc resection of the frontal bone including the mass was performed, and the circumferential normal bone tissue was resected. The frontal bone was removed (Fig. 2B) with care taken not to damage the frontal sinus mucosa. The frontal sinus was sealed with a collagen patch (Tachocomb) and a cranioplasty was performed using bone cement. A silastic drain was inserted and the wound was sutured layer by layer with 6-0 Monocryl and 6-0 nylon. Histological examination revealed an intraosseous hemangioma in the right frontal sinus bone (Fig. 3). The resection margin was clear. At 6 months postoperative, a clean wound was confirmed without any com-
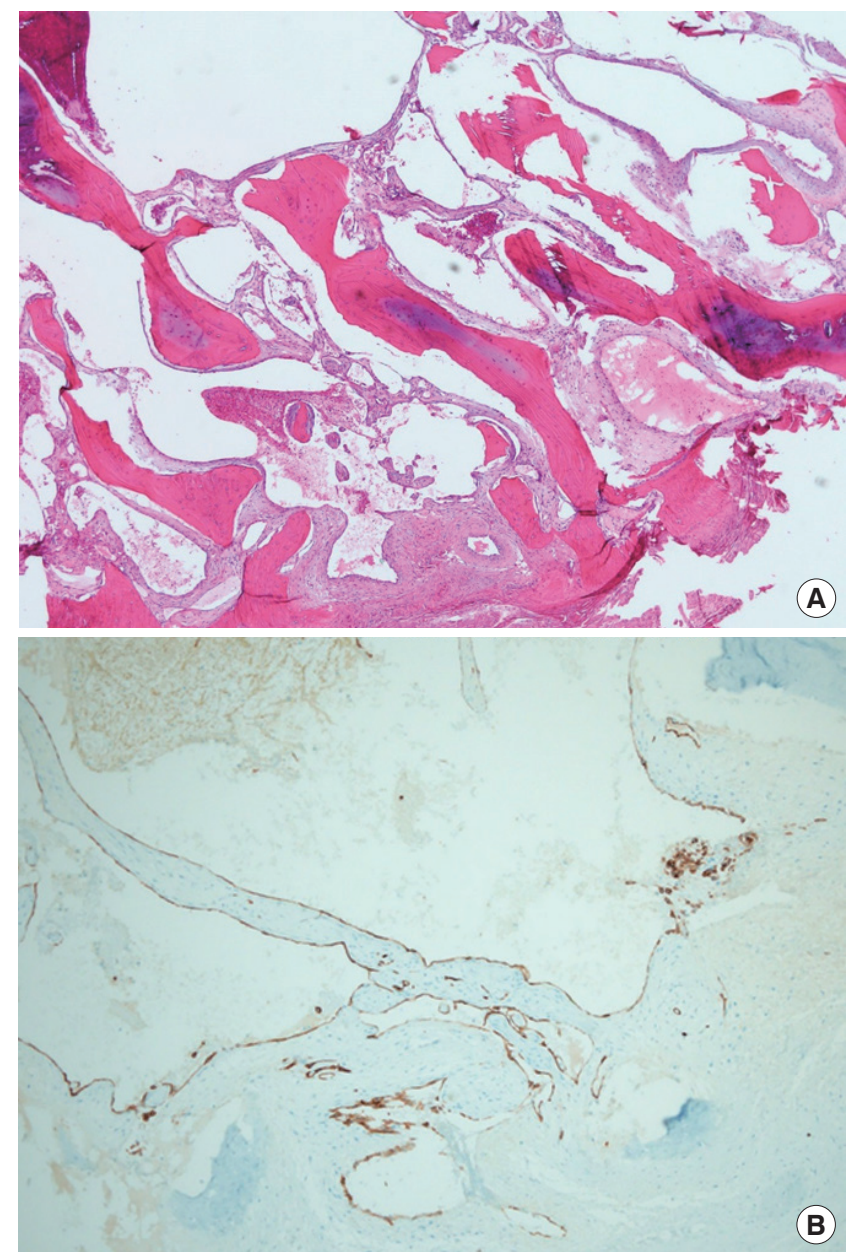

Fig. 3. Histologic images. (A) Intraosseous hemangioma ( $\mathrm{H} \& \mathrm{E}, \times 40)$ : cavernous hemangioma showing extended, thin-walled vessels, and sinuses lined by a single layer of endothelial cells. (B) Immunohistochemistry staining for CD34 revealed blood vessel formation $(\times 100)$ : CD34 highlights the contours of the malformed vessels and along lining endothelial cells.

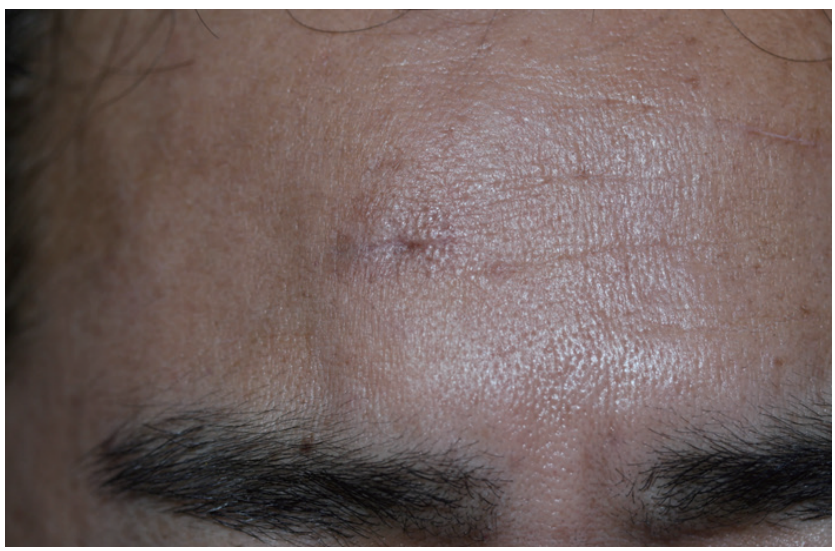

Fig. 4. Six-month postoperative photograph.

plications, and there was no local recurrence (Fig. 4). 


\section{DISCUSSION}

Osteoma is most commonly suspected in cases of firm and immobile forehead masses. However, the possibility of various pathologies other than osteoma, such as intraosseous hemangioma, fibrous dysplasia, Langerhans cell histiocytosis, multiple myeloma, meningioma, eosinophilic granuloma, and metastatic carcinoma, must also be considered [4]. Preoperative CT is essential for making an accurate preoperative differential diagnosis, planning for precautions, and establishing a surgical plan that can minimize postoperative complications [6,7]. Additionally, the incision can be minimized by confirming the mass's exact invasiveness and size using preoperative $\mathrm{CT}$, producing better aesthetic results. No special contrast is required for preoperative CT [6].

If suspected intraosseous hemangioma of the forehead is first differentiated through CT, the intraosseous hemangioma is recommended to be observed without further treatment because it is benign. Treatment is needed when symptoms of mass occur, when bleeding occurs, or when cosmetic problems occur [8]. The preferred treatment is complete surgical resection within an adequate margin of normal healthy bone [8]. To successfully perform this treatment, it is common to remove the mass using craniotomy via a bicoronal incision. When an intraosseous hemangioma has expanded bidirectionally, since the inner surface of the mass may be adherent to the dura mater, it is necessary to secure a sufficient field of view using the bicoronal incision to enable careful dissection between the tumor and the dura. However, in this case, the intraosseous hemangioma had a small diameter $(1.5 \mathrm{~cm})$ and did not require an extensive craniotomy since it did not involve the dura. When removing intraosseous hemangioma through a direct small skin incision, as in this case, the use of a burr enables the identification of normal bone tissue, but care must be taken to prevent tearing the dura or the frontal sinus mucosa. If the mass is small and does not invade the dura, the possibility of surgical access through a direct small skin incision can be inferred. However, to date, the criteria for choosing between direct small skin incision and the bicoronal incision have not been elucidated. Further research is needed to answer this question.

The use of a bicoronal incision for removing the intraosseous hemangioma of the frontal bone provides extensive surgical access to the upper and middle facial thirds, enabling a smooth operation. However, access via a bicoronal incision is aggressive, and complications such as postoperative hypoesthesia, partial unilateral frontal motor deficit, infection, hypertrophic scars, varying degrees of alopecia, seroma, or hematoma in the immediate postoperative period can occur [9]. Accordingly, when performing surgery in the upper and middle facial thirds, sufficient examination should be performed preoperatively, which will prevent overuse of the bicoronal incision approach.

Cranialization, the procedure for patients in whom communication between the frontal air sinus and the outside space is blocked, and the air sinus space is integrated with the intracranial space, may be necessary when removing bone tumors in volving the frontal sinus $[10,11]$. Postoperative complications after frontal sinus mass excision, including cerebrospinal fluid leakage, rhinorrhea, meningitis, and wound infection may occur in cases of frontal sinus cavity mucosal tearing or injury to the posterior wall of the frontal sinus or nasofrontal duct [1214]. Cranialization is essential for preventing these complications.

A bicoronal incision must be used to ensure smooth frontal sinus cranialization since it allows the harvesting of a pericranial flap and calvarial bone grafts. As in this case, when the surgical plan was determined before mass removal, the postoperative frontal sinus mucosa, posterior wall of the frontal sinus, and nasofrontal duct appeared intact, eliminating the need for cranialization [11]. If cranialization is unnecessary, it may be better to use a direct small skin incision instead, which may be cosmetically favorable.

One of the disadvantages of direct skin incision is that it is difficult to secure sufficient intraoperative view for resection. Hence, the operation may end without the grossly normal bone level being accurately identified after the resection of the frontal area bone tumor. Since this can cause intraosseous hemangioma recurrence, long-term follow-up is necessary to monitor for recurrence.

All patients undergoing reconstructive surgery of the frontal sinus can develop delayed complications [12]. Therefore, patients must fully understand the complications that may occur and visit the hospital as soon as possible in such cases.

Surgical excision of intraosseous hemangioma in the frontal sinus bone can be performed via direct incision or the bicoronal approach. In this case, the direct incision approach was used to achieve smaller scars and faster recovery than the bicoronal approach. However, to date, the criteria for choosing between direct small skin incision and the bicoronal incision have not been elucidated; therefore, future studies are required.

\section{NOTES}

\section{Conflict of interest}

No potential conflict of interest relevant to this article was reported. 


\section{Ethical approval}

The study was approved by the Institutional Review Board of Chung-Ang University Hospital (IRB No. 2010-008-19336) and performed in accordance with the principles of the Declaration of Helsinki. Written informed consent was obtained.

\section{Patient consent}

The patient provided written informed consent for the publication and the use of his images.

\section{ORCID}

Hyeon Seok Kim https://orcid.org/0000-0002-0142-9314

Woo Seob Kim https://orcid.org/0000-0002-4104-3926

Han Koo Kim https://orcid.org/0000-0002-2849-3973

Tae Hui Bae https://orcid.org/0000-0002-0342-1439

\section{Author contribution}

Conceptualization, data curation: WSK, HKK, THB. Formal analysis, visualization: HSK. Supervision: THB. Writing - original draft: HSK. Writing - review \& editing: WSK, HKK, THB.

\section{REFERENCES}

1. Choi JS, Bae YC, Kang GB, Choi KU. Intraosseous hemangioma of the orbit. Arch Craniofac Surg 2018;19:68-71.

2. Kim JH, Ahn CH, Kim KH, Oh SH. Multifocal intraosseous calvarial hemangioma misdiagnosed as subgaleal lipoma. Arch Craniofac Surg 2019;20:181-5.

3. Cheng NC, Lai DM, Hsie MH, Liao SL, Chen YB. Intraosseous hemangiomas of the facial bone. Plast Reconstr Surg 2006;117: 2366-72.

4. Seo BF, Kang KJ, Jung SN, Byeon JH. Skeletal cavernous hemangiomas of the frontal bone with orbital roof and rim involve- ment. Arch Craniofac Surg 2018;19:214-7.

5. Hahn HM, Lee YJ, Park MC, Lee IJ, Kim SM, Park DH. Reduction of closed frontal sinus fractures through suprabrow approach. Arch Craniofac Surg 2017;18:230-7.

6. Brumm JD, Paul BC, Miller PJ. Evaluation and excision of firm, immobile forehead masses. Am J Cosmet Surg 2015;32: 59-65.

7. Balan P, Gogineni SB, Shetty SR, Areekat FK. Radiologic features of intraosseous hemangioma: a diagnostic challenge. Arch Med Health Sci 2014;2:67-70.

8. Park BJ, Lim SY, Park JH, Pyon JK, Mun GH, Bang SI, et al. Intraosseous hemangioma of the zygoma and its management. J Korean Soc Plast Reconstr Surg 2008;35:735-8.

9. Lee Y, Choi HG, Shin DH, Uhm KI, Kim SH, Kim CK, et al. Subbrow approach as a minimally invasive reduction technique in the management of frontal sinus fractures. Arch Plast Surg 2014;41:679-85.

10. Lotfinia I, Shokouhi G, Salehpoor F, Baboli S, Totongee J. Evaluation of cranialization technique in the treatment of frontal sinus fractures. Med J Islam Repub Iran 2005;19:231-6.

11. Pollock RA, Hill JL Jr, Davenport DL, Snow DC, Vasconez HC. Cranialization in a cohort of 154 consecutive patients with frontal sinus fractures (1987-2007): review and update of a compelling procedure in the selected patient. Ann Plast Surg 2013;71:54-9.

12. Kim YW, Lee DH, Cheon YW. Secondary reconstruction of frontal sinus fracture. Arch Craniofac Surg 2016;17:103-10.

13. Yavuzer R, Sari A, Kelly CP, Tuncer S, Latifoglu O, Celebi MC, et al. Management of frontal sinus fractures. Plast Reconstr Surg 2005;115:79e-93e.

14. Oh SY, Choi JS, Lim JS, Kim MC. Eyeball deviation by orbital mucocele after midface sinus injury. Arch Craniofac Surg 2020; 21:53-7. 